\title{
Collaborative Solving in a Human Computing Game Using a Market, Skills and Challenges
}

\author{
Olivier Tremblay-Savard \\ University of Manitoba \\ Winnipeg, Canada \\ tremblao@cs.umanitoba.ca
}

\author{
Alexander Butyaev \\ McGill University \\ Montreal, Canada \\ butyaevaa@gmail.com
}

\author{
Jérôme Waldispühl \\ McGill University \\ Montreal, Canada \\ jeromew@cs.mcgill.ca
}

\begin{abstract}
Crowdsourcing with human-computing games is now a wellestablished approach to help solving difficult computational problems (e.g. Foldit, Phylo). The current strategies used to distribute problems among participants are currently limited to (i) delivering the full problem to every single user and ask them to explore the complete search space (e.g. Foldit), or (ii) decomposing the initial problem into smaller sub-problems and aggregate the solutions returned by gamers (e.g. Phylo). The second approach could be used to explore larger search spaces while harnessing collective intelligence, but popular crowdsourcing systems making use of the Amazon Mechanical Turk deliberately forbid communication between participants to avoid group-think phenomena. In this paper, we design a novel multi-player game-with-a-purpose, and analyze the impact of multiple game mechanisms on the performance of the system. We present a highly collaborative human-computing game that uses a market, skills and a challenge system to help the players collectively solve a graph problem. The results obtained during 12 game sessions of 10 players show that the market helps players to build larger solutions. We also show that a skill system and, to a lesser extent, a challenge system can be used to influence and guide the players towards producing better solutions. Our collaborative game-with-a-purpose is open-source, and aims to serve as a universal platform for further independent studies.
\end{abstract}

\section{ACM Classification Keywords}

H.5.3 Group and Organization Interfaces

\section{Author Keywords}

Game-with-a-purpose; Human computing; Collaboration; Crowdsourcing; Graph problem; Market; Trading game; Skills; Challenges.

\section{INTRODUCTION}

Human-computation and crowdsourcing are now perceived as valuable techniques to help solving difficult computational problems. In order to make the best use of human skills in

Permission to make digital or hard copies of all or part of this work for personal or classroom use is granted without fee provided that copies are not made or distributed for profit or commercial advantage and that copies bear this notice and the full citation on the first page. Copyrights for components of this work owned by others than the author(s) must be honored. Abstracting with credit is permitted. To copy otherwise, or republish, to post on servers or to redistribute to lists, requires prior specific permission and/or a fee. Request permissions from Permissions@ acm.org.

CHI PLAY '16, October 16 - 19, 2016, Austin, TX, USA

Copyright is held by the owner/author(s). Publication rights licensed to ACM ACM 978-1-4503-4456-2/16/10\$15.00

DOI: http://dx.doi.org/10.1145/2967934.2968104 these systems, it is important to be able to characterize the expertise and performance of humans as individuals and even more importantly as groups. Currently, crowd-computing approaches make use of popular platforms such as Amazon Mechanical Turk (AMT) [1, 24] or Crowdcrafting [21]. The initial problem is decomposed into smaller sub-tasks that are distributed to individual workers and then aggregated to build a solution. This is also the case in popular scientific humancomputing games such as Phylo [12, 18]. Importantly, these systems prevent any interaction between workers in order to prevent groupthink phenomena and bias in the solution [22]. However, such constraints are necessarily limiting the capacity of the system to harness the cognitive power of crowds and make full benefit of collective intelligence. For instance, iterative combinations of crowdsourced contributions can help enhancing creativity [30]. Similarly, the presence of a broad spectrum of expertise in a crowdsourcing community has been shown to increase innovation and the advance of knowledge in the group [2]. The usefulness of parallelizing workflows has also been suggested for tasks accepting broad varieties of answers [20].

The benefits of developing recommendation systems or coordination methods in collaborative environments has been demonstrated [15, 3, 31]. Therefore, in order to gain expressivity and improve their performance, the next generation of human-computation systems will certainly need to implement mechanisms to promote and control the collaboration between workers. Nonetheless, before transitioning to this model, it is important to first estimate the potential gains in productivity, and quantify the usefulness of the mechanisms and incentives to promote collaborative solving and prevent groupthink. A first step in this direction was made recently: mathematical models have been used to simulate the interactions between the participants of crowdsourcing projects in order to estimate the optimal group size depending on the difficulty of the problem [5], and analyze the effect of repeated encounters on the reactive strategies of competing groups that can attack and sabotage each other [23].

Computer games are now a widely used and effective media to complete crowdsourcing tasks [16]. Multiple studies already investigated the impact of design on human-computing tasks $[17,10,11]$. Incidentally, they are also an excellent and potentially powerful framework to study collaborative work.

Historically, computation on graphs has proven to be a good model to study the performance of humans in solving com- 
plex combinatorial problems [14]. Experiments have been conducted to evaluate the dynamics of crowds collaborating at solving graph problems [13] but still, little is known about the efficiency of the various modes of interaction.

In this paper, we propose a formal framework to study human collaborative solving. We embed a combinatorial graph problem into a novel multiplayer game-with-a-purpose [27, 7], which will be used to engage participants and analyze collective performances. More precisely, we design a market game in which players can sell and buy solutions or bits of information, and couple this platform with (i) a skills system to enhance the efficiency of specific gaming strategies and (ii) a challenge system to guide the work of the crowd.

Most existing simultaneous collaborative games rely on two or three players trying to agree on an annotation of an image [27, $8,28]$, a song [29, 19], a video [6] or a text [4]. In other crowdsourcing projects, collaboration between workers is iterative $[26,25,9]$. To the best of our knowledge, it is the first time that a market system is proposed to allow simultaneous collaborative solving by a large group of people.

We developed this market game to investigate the validity of the following hypotheses.

\section{Hypotheses}

The development of the game with its three main features, i.e. the market, the skills and the challenge system, was based on those four hypotheses:

1. A market system will help the players build longer solutions.

2. A skill system is useful to orient the players into doing specific actions that are beneficial to the game and other players.

3. A challenge system is effective in encouraging the players to do a specific action in the game.

4. The collected solutions are larger when all the three features are present in a game session, independently of the players' personal skills.

To test those hypotheses, we conducted a study on 120 participants using different variants of our market game. Our results confirm the benefits of using a trading platform to produce better solutions. Interestingly, we also found that a skills system helps to promote actions that are favorable to the collective solving process, but that the efficiency of a skill is reduced if it is designed to help solve one of the primary objectives of the game. Finally, we observed that a precise parametrization of challenges (i.e. finding an appropriate difficulty, nor too easy, nor too difficult) is required to result in an improvement of the quality of the collective work.

Our game is freely available at http://csb.cs.mcgill.ca/market-computing, and can be used as a platform for further independent studies.

\section{PROBLEM}

The game was implemented to solve a graph problem, which is the problem of finding maximal cliques in a multigraph. In simpler terms, the goal is to find subsets of vertices in the graph such that any two distinct vertices in the subsets are connected. In the case of this problem, we are dealing with a multigraph, which is simply a graph that can have multiple parallel edges. Let $G(V, E)$ be a multi-colored graph, where each vertex $v \in V$ has a set of colors $c(v)$. There is a colored edge $e=(v, u) \in E$ between the vertices $v$ and $u$ for every color in $c(v) \cap c(u)$ (i.e., one for every color that they have in common). In other words, there is no colored edge between two vertices $v$ and $u$ for which $c(v) \cap c(u) \neq \emptyset$. Let $|C|$ be the total number of colors in the graph. The problem is then the one of finding maximal cliques for each possible $n$ number of colors (where $1 \leq n \leq|C|$ ), i.e. cliques in which all the edges (and vertices) have the same $n$ colors. A simple exact algorithm can solve the problem in $O\left(|V| 2^{|C|}\right)$. We make the conjecture that it is also the worst time complexity of the problem.

This problem was chosen for two reasons. First, it can be solved quickly by a computer when the number of colors is small, thus making it possible to compute the exact solution and measure the percentage of the solution that is found by the players in a game session. Second, this problem can easily be translated into a color matching game, which takes advantage of the ability of human perception. Indeed, since the colored edges between the vertices are given implicitly by the colors of the vertices, it is possible to show the players only the colored vertices. To solve the problem, the players have to find the largests sets of circles with colors in common, for all possible subsets of colors.

Note that it is not our goal to compare the performance of players with the performance of computers in solving this problem. With a limited number of colors (like six in our tests), the exact algorithm can solve the problem in seconds. For this study, we required a problem that was structured enough so that we could easily calculate the optimal solution and evaluate the performance of the players depending on what features were on or off and also the effect of the different features on the quality of solutions.

\section{PRESENTATION OF THE GAME}

\section{Goal of the game}

The main objective of the game is to build sequences (i.e. sets) of circles (circles represent vertices of the graph) that (i) are as long as possible and (ii) contain as many colors in common as possible. Circles used by the players to build the sequences either come random packages bought from the system or they come from another player through the market. The sequences can then be sold to the system for a certain amount of game money, which is determined by a scoring function that takes into account the length and the number of colors in common of the sequence.

The players continously have to fill up their "hand" by buying circles, create the best sequences of circles from their hand and sell them to the system for in-game money. Figure 1 shows the gameplay loop and how the inclusion of the market system and challenges affect it.

\section{Scoring function}

The score of a sequence sold to the system is equal to baseScore $_{n} *$ seqLength $^{2}$, where baseScore ${ }_{n}$ is a base score 


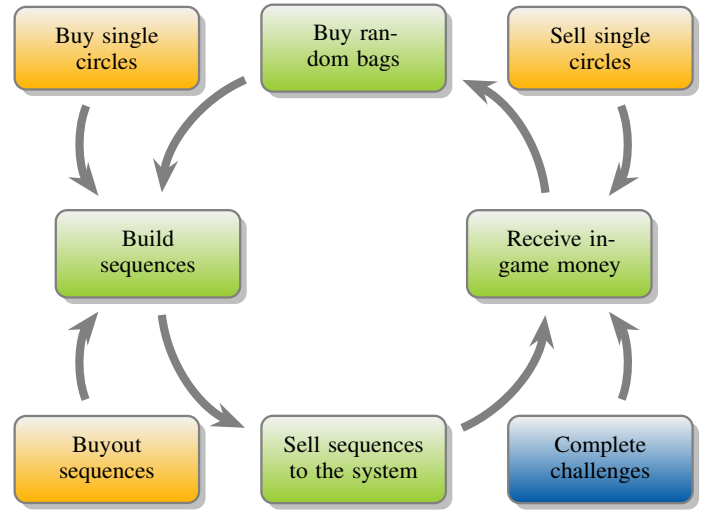

Figure 1. Gameplay loop diagram. The green boxes represent the actions that the players can make when there are no challenges and no market. The orange (resp. blue) boxes represent the actions that are allowed when the market (resp. challenge system) is present and how they interact with the gameplay.

depending on the number of colors in common (see Table 1) and seqLength is the length of the sequence. The base scores were calculated based on the exact solution for the graph that was generated for the tests (see section Generating the graph for a description of the graph that was used) in such a way to give a reward that is proportional to the difficulty of building the sequence. More precisely, we calculated the average length $L_{n}$ of all solutions for each $n$ number of colors. The base score is simply the reciprocal of this average $\left(1 / L_{n}\right)$ multiplied by a balancing factor (505 in our case). The balancing factor was chosen in order to get a score of 500 for a sequence of length 10 with only one color in common, which is exactly the price of two random packages of circles. Also notice that the value of a sequence is exponential in relation to its length, which is to encourage players to build the longest possible sequences.

\begin{tabular}{cccccccc}
\hline Number of colors & 0 & 1 & 2 & 3 & 4 & 5 & 6 \\
Base score & 0 & 5 & 14 & 26 & 40 & 55 & 72 \\
\hline
\end{tabular}

Table 1. Value of the base score depending on the number of colors in common

The idea behind the scoring function is simple. Building simple short sequences with few colors in common can be done very quickly without much effort, so the score of those sequences is low. On the other hand, building long sequences with a lot of colors in common takes longer, so a high score is given to those sequences. Hence there is a tradeoff for the players between creating and selling many simple sequences, or creating and selling fewer more complex sequences.

\section{Game interface}

The game client and the server were built in Java 1.7. As shown in Figure 2, the game interface can be divided into 3 parts: the player information panel, the game panel and the market panel.

\section{A: Player information panel}

This panel simply contains information on the player's wallet, the current level of the player and has three buttons, allowing the player to open dialogs showing information on the current challenge, the skills (see section Skills for a description of the available skills) and the leaderboard. One experience point is given to the player for each game dollar that he/she wins. The player can lose game money, but cannot lose experience points (experience points can only go up). Every time a player levels-up, he/she gets a skill point that can be used to improve any of the skills.

\section{B: Game panel}

The first component of the game panel is the 'My sequence' panel, which shows the current sequence that is being built by the player. The maximum size of a sequence is 10 . Colors in common in the sequence are indicated by a thick black border surrounding the colors in the circles. Players can use the arrows to switch between the different sequence slots ( 2 sequence slots are available at the start of the game). The current value of the sequence is shown at the right, and the price for adding one more circle with the same colors in common is shown right below in gray. Finally, the sell button allows the player to sell the current sequence to the system: the sequence then disappears and the money is given to the player. Selling a sequence is equivalent to submitting a solution to the system.

The second component is the 'My hand' panel, which can contain up to 20 circles. Players can add a circle to the sequence by clicking on it. Circles are represented by their colors and by a price label (in a black box). The price corresponds to the current value of the circle on the market. Clicking on the price label sells the circle to the highest bidder on the market. Circles that are bought from a random package or from other players are sent to the hand.

The 'Awaiting to get sold' is where the circles are sent just before being sold to the highest bidder. If the bid disappears before the transaction is completed, the 'sold' circle will stay there. The player can then click on it to cancel the selling and put it back in the hand.

Finally, the bottom panel is a news feed, showing information on the game state, like the remaining time to complete the challenge and the last transactions completed by the player.

\section{C: Market panel}

At the top of the market panel, buttons allow the player to create bids for circles or to buy random packages (or bags) of circles. The 'Random bag' costs $\$ 250$ and contains 5 circles with fewer colors. The 'Premium bag' costs $\$ 500$ and contains 5 circles with a higher chance of getting circles with many colors.

Right below the buttons is the 'Automatic bids' panel, which allows the player to get automatic bids for circles corresponding to the sequences that he or she is building. A percentage of profit for the price of the automatic bids can be set with the slider. The profit is defined as the money the player would make by adding one more circle with the same colors in the current sequence (difference between the gray and black prices above the Sell button).

The 'My bids' panel shows all the bids that the player currently has on the market. The bid price is shown below the circle (in the black box). On the right side of the circle is the number of sequences with the same colors that the player can buy from 
other players (in the blue box). Clicking on the blue box opens a window showing the list of sequences that can be bought. Buying a sequence from another player is called a 'buyout' (see the following subsection for a more detailed description of buyouts).

Finally, the last panel at the bottom shows the last circle or sequence that was bought by the player.

\section{Market}

The market has three functions: (i) allow the players to exchange circles through a bidding system, (ii) allow players to buy sequences built and sold by other players so that they can be improved, and (iii) merge together sequences of length 10 to create super circles that are then put back in the game.

For every subset of colors, the server has a list of all the bids that are currently on the market. The value of the highest bid on the market is shown below every circle under the possession of the players. When a circle is sold by a player, it is sent through the server to the highest bidder.

Buyouts work differently. Players cannot bid on sequences, but the server holds for two minutes all the sequence that have been sold by the players. During those two minutes, other players can buy the sequences for a price that is equal to $150 \%$ of the initial score of the sequence. When a buyout is made, the bonus game money is sent to the player who initially sold the sequence to the system.

Finally, the game system creates a super circle every time a sequence of length 10 is sold by a player. A super circle of level 2 (representing 10 circles) counts as two circles when put in a sequence. Super circles can be of any level (a sequence of 10 super circles of level 2 form a super circle of level 3 , and so on). The idea behind the creation of the super circles was to remove the limitation of the maximum sequence size imposed by the game interface.

\section{Skills}

Four different skills were implemented in the game. One skill point is awarded to a player when he or she levels up, which can then be put in any of the four skills. The maximum level of each skill is six (there are six levels of bonuses). Each skill was put in the game as a way to guide the player in doing actions that are beneficial to the system or to the other players:

- Buyout King: lowers the price of buying a sequence from another player (goal: encourage buyouts);

- Color Expert: gives a bonus to selling sequences that have more than one color in common (goal: push players to build more multicolored sequences);

- Sequence Collector: gives an additional sequence slot (goal: give more space to encourage the creation of longer sequences with more colors in common);

- Master Trader: gives a bonus to selling circles to other players (goal: promote the selling of individual circles).

\section{Challenge system}

We implemented a challenge system that analyzes the recent actions of the players and creates a new challenge every five minutes. The five challenge types are:
- Sell/buy circles: requires the players to sell or buy circles;

- Buyout sequences: requires the players to buy sequences from other players;

- Minimum number of colors: requires the players to sell sequences with at least a certain number of colors in common;

- Minimum sequence length: requires the players to sell sequences with a minimum sequence length;

- Specific colors in common: requires the players to sell sequences with a specific subset of colors in common.

Basically, the system continuously monitors the activities of the players and decreases or increases the probabilities of each challenge type. The next challenge is then selected using a multinomial sampling on these probabilities. The number of times $T$ that the challenge-related action must be completed is selected randomly between 2 and 4 . The prize that is awarded for completing the challenge is equal to $\$ 1500 * T$.

\section{EXPERIMENTS}

\section{Independent and dependent variables}

In the context of this study, there were three independent variables: the market (present; not present), the skills (present; not present) and the challenges (present; not present). Instead of trying all 8 possible combinations of independent variables, we decided to focus on four game conditions:

1. All features present (or A)

2. Everything except the market, hereafter referred to as "No Market" (or NM)

3. Everything except the skills, hereafter referred to as "No Skills" (or NS)

4. Everything except the challenges, hereafter referred to as "No challenges" (or NC)

Focusing on those four playing conditions allowed us to repeat each experiment more times with different groups of players. Moreover, the goal was to evaluate the importance of every game feature by removing them one at a time and evaluating the effect on the results obtained by the players.

As for the dependent variables, we were interested in measuring the following:

1. Percentage of the problem solved

2. Total experience points earned by the players

3. Average sequence length of the sequences created by the players $\left(\sum_{i=1}^{T S} s L_{i} / T S\right)$

4. Average number of colors in common of the sequences created by the players $\left(\sum_{i=1}^{T S} n b C_{i} / T S\right)$

5. Proportion of sequences of more than one color in common created by the players $((T S-T 1 C) / T S)$

6. Number of circles sold individually to another player

7. Number of sequences bought from other players (buyouts)

where $T S$ is the total number of sequences sold by all players during a session, $s L_{i}$ is the sequence length of sequence $i, n b C_{i}$ is the number of colors in common of sequence $i$ and $T 1 C$ is the total number of sequences of one color sold by all players during a session. 


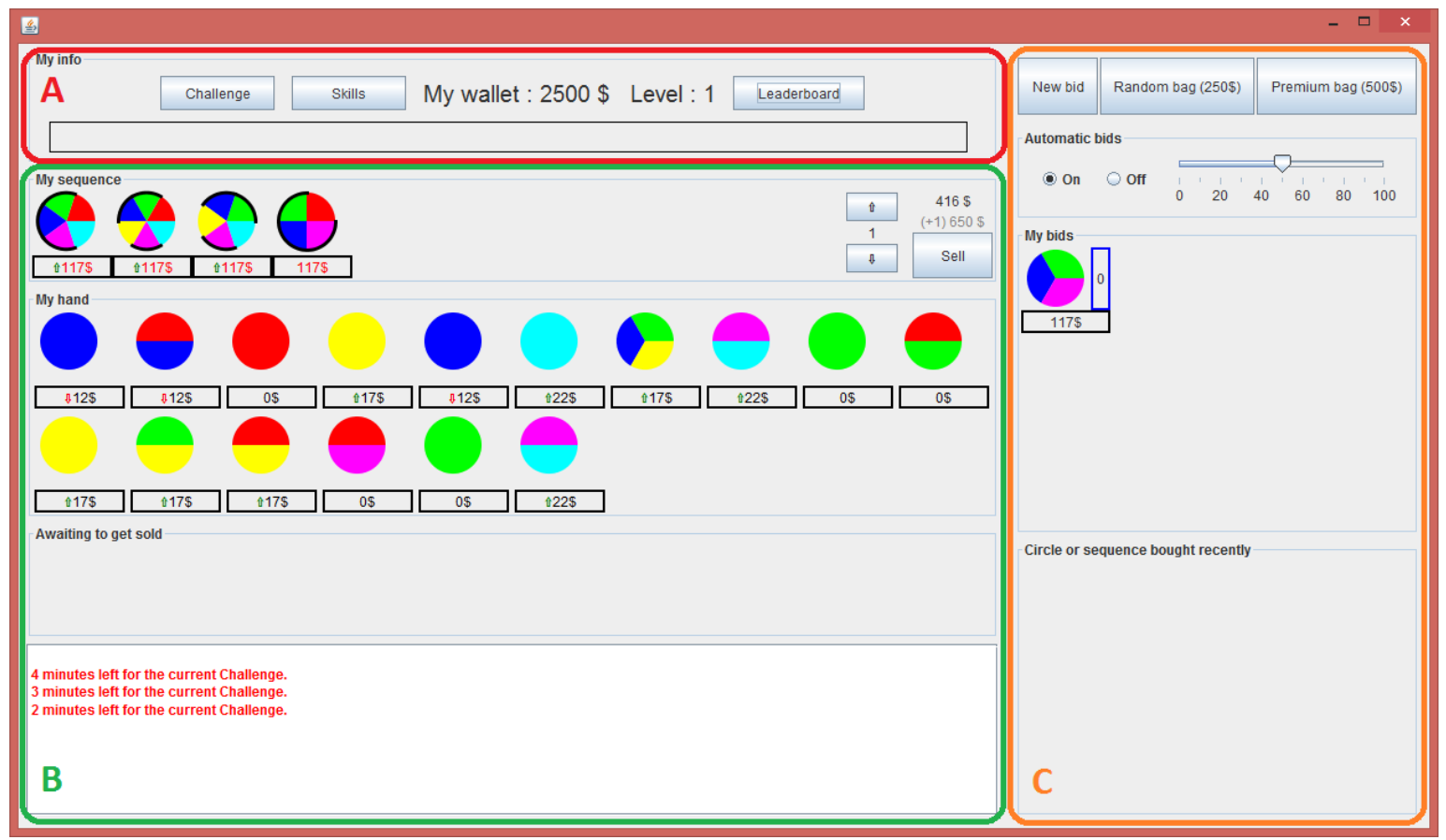

Figure 2. The game interface, separated in three panels. Panel A (inside the red box) is the player information panel. Panel B (inside the green box) is the game panel. Panel C (inside the orange box) is the market panel.

\section{Game sessions}

We recruited 120 people in total to test our game. Out of those 120 participants, $35 \%$ were female and $65 \%$ were male. Their average age was 25.7 and average video gaming time per week was 4.7 hours. Roughly $75 \%$ of the participants were undergrad students from McGill University and University of Montreal. The other 25\% were grad students and volunteers recruited through social media. Out of all the participants, about half of them were currently studying or working in computer science.

We divided the participants into groups of 10 and repeated three times each of the four game conditions presented in the previous subsection. Each participant was playing the game for the first time, except for some people that were invited as replacements to deal with last minute cancellations. Before starting each game session, the players were shown a document explaining the rules of the game and the interface. The participants were told that they were playing a human computing game that aims to solve a graph problem, but the mathematical problem in question was not described to the players. They were also asked to fill in a questionnaire so that we could get information on the participants, such as their age, their abilities at puzzle solving and their experience with video games for example. For all the experiments, the game session lasted 45 minutes.

\section{Generating the graph}

We generated one random colored multigraph that we used for all the 12 tests. Since the edges in the graph depend entirely on the colors of the vertices, it is sufficient to generate only the colored vertices. For the tests, a graph containing 300 vertices and 6 different colors was generated. To randomly select the number of colors for each vertex, a geometric distribution of parameter $p=0.5$ was used, so that the vertices with a lot of colors are rarer. Once the number of colors was selected for the vertex, the set of colors was selected uniformly.

\section{RESULTS AND DISCUSSION}

\section{Testing hypothesis 1: the efficiency of the market}

The market system we implemented in the game allows the players to exchange circles and partial solutions (in the form of buyouts). The main goal of the market is to help the players in building longer sequences.

As shown in Figure 3, the three game sessions in which we had the lowest average of sequence lengths (for all the sequences sold by all the players) are the ones that were played without the market, with averages of 4.40 for NM, 4.19 for NM-2 and 4.63 for NM-3. Even if we consider the super circles (the special circles that are actually 10 circles combined into one), the average sequence lengths for those three sessions are still the lowest ones, with values of 4.90 for both NM and NM-2, and 5.40 for NM-3.

Since the distribution of the lengths for all the sequences sold to the system during a game session do not follow a normal distribution, we used a non-parametric test (Kruskal-Wallis) to verify if the sequence lengths of the different game sessions seem to come from the same distributions. The KruskalWallis test revealed a significant effect of the game conditions on the sequence lengths without considering super circles $\left(\chi^{2}(11)=1391.7, p<2.2 E-16\right)$ and also when considering super circles $\left(\chi^{2}(11)=1388.4, p<2.2 E-16\right)$. 


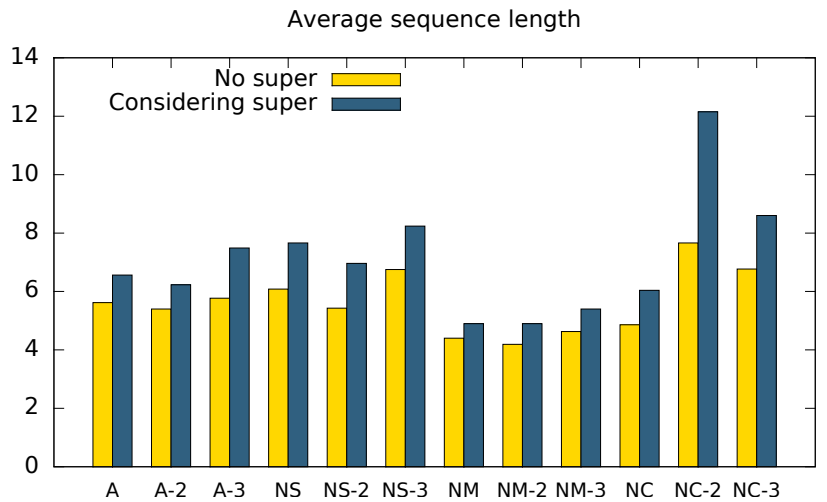

Figure 3. Average sequence length for every game session, not considering the super circles and considering the super circles (e.g. a super circle of level 2 in a sequence represents 10 circles in the solution). ' $A$ ', 'A-2' and 'A-3' represent the tests with all the features on; 'NS', 'NS2' and 'NS-3' represent the tests without the skills; 'NM', 'NM-2' and 'NM-3' represent the tests without the market; 'NC', 'NC-2' and 'NC-3' represent the tests without the challenges.

We then made a post hoc test (Dunn's test) to do pairwise comparisons between all the groups. With or without considering super circles, all the game conditions were shown to be significantly different $(p<0.01)$, except a few shown in table 2 . Note that the strongest similarities are found between the three 'All' groups and the three 'No market' groups. Some of the 'No skills' experiments are found to be similar to the 'All' groups, which could indicate that the presence of the skills have a very limited effect on the sequence length. The NC experiment is found to be similar to two 'No market' groups, but that can be explained by the fact the players for the NC experiment were very weak (as can be seen by the total experience gained during that session in Figure 10).

Note that even in the two sessions (A and NC) for which we had the smallest total experience (which is a way to evaluate the strength of the players; see Figure 10), the averages of sequence lengths were higher than the ones of the 3 game sessions without the market, even though we had some very strong groups for NM-2 and NM-3 (as shown by the total XP in Figure 10). Those observations confirm that the market is helping the players in the creation of longer sequences.

\begin{tabular}{c|cccccccc}
\hline & A-2 & A-3 & NS & NS-2 & NS-3 & NM & NM-2 & NM-3 \\
\hline A & n/s & n & & n/s & & & & \\
A-2 & & $n$ & & n/s & & & & \\
A-3 & & & n/s & & & & & \\
NC & & & & & & $n$ & & $n / s$ \\
NC-3 & & & & & n/s & & & \\
NM & & & & & & & n/s & n \\
\hline
\end{tabular}

Table 2. Similar groups of sequence length distributions, as reported by Dunn's test. An 'n' in the table represents a similar pair when not considering super circles, and an 's' in the table represents a similar pair when considering super circles.

\section{Testing hypothesis 2: the benefits of a skill system}

We implemented the skill system for two reasons: (i) to give the players more incentive to accumulate experience points as fast as possible, because the reward for leveling-up is an additional skill point, and (ii) to influence indirectly the players into doing actions that are either improving the solutions collected by the system or helpful to the other players (which in the end will also improve the solutions). In our game, two skills were related to the market (Buyout King and Master Trader) and two skills were related to building sequences (Color Expert and Sequence Collector). In the following paragraphs, we will analyze how those four skills affected the strategies and actions of the players. Note that when some players lost all their money in the game, they had to start a new game. In our results, we count both new games as if they were played by different players, since the players who restart might choose a different set of skills the second time. That explains why the total number of players is larger than 120. Players of the 'No skills' game condition were considered and put automatically in the without skill group.

\section{Buyout King}

The Buyout King skill allows the players to reduce the price of buying a sequence from another player (which we call a buyout). The idea behind this skill was to encourage the players to buy small sequences built by other players so that they could improve them before selling them back to the system. In other words, a buyout is the action of buying a partial solution made by another player in order to improve it.

Figure 4-left shows statitics for the players who have put at least one skill point in the Buyout King skill and the players who did not use the skill at all. We were interested in the number of buyouts that the players with the skill were making compared to the rest of the players. Note that since this skill is related to the market, we did not consider the 'No market' sessions for these results.

The median value for players who spent a skill point in the Buyout King skill is 15, while the median value for the players without the skill is 1.5 , indicating that half of the players without the skill did not use the buyout at all or used it only once. Since the distribution of the number of buyouts is not following a normal distribution (the Shapiro-Wilk test rejected the null hypothesis with $p=2.6 E-10$ ), we used a MannWhitney's U test to compare the medians of the two groups. We found a significant effect of the presence of this skill on the medians $(U=1629.5, p=0.004$, effect size $r=0.28)$.

\section{Master Trader}

The Master Trader skill allows the players to get bonus money in addition to the regular market price for each circle they sell individually. This skill was put in the game in order to increase the activity on the market by encouraging more players to send the circles that they don't need to players who need it the most.

Figure 4-right shows statistics for the players who have put at least one skill point in the Master Trader skill and all the other players. We were interested in comparing the number of individual circles that were sold to another player for the two different categories. Once again, since this skill depends on the presence of the market, we did not consider the 'No market' experiments in the results shown. 

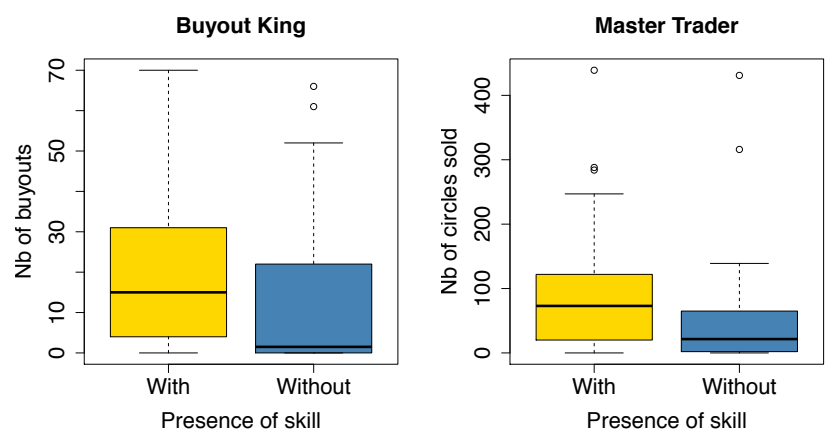

Figure 4. Left: Boxplot of the number of buyouts made by players with (37 players) and without (66 players) the Buyout King skill. Right: Boxplot of the number of circles sold individually by players with ( 33 players) and without (70 players) the Master Trader skill.

The median value for the players who had selected the Master Trader skill (73) is more than three times larger than the one for the rest of the players (21.5). Since the distribution of the number of circles sold individually is not following a normal distribution (the Shapiro-Wilk test rejected the null hypothesis with $p=5.3 E-13$ ), we used a Mann-Whitney's $\mathrm{U}$ test to compare the medians of the two groups. We found a significant effect of the presence of the Master Trader skill on the medians $(U=1633.5, p=7.2 E-4$, effect size $r=0.33)$.

\section{Color Expert}

The Color Expert skill gives a bonus multiplier to the scoring function for sequences with more than one color in common. This skill was implemented in order to give extra motivation to build sequences with many colors in common, since they are harder to build. Indeed, more focus is needed from the player to match many circles with more than one color in common.

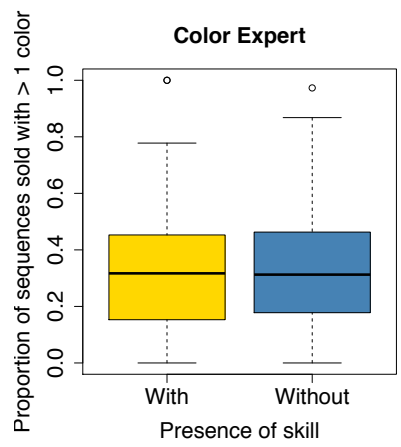

Figure 5. Boxplot of the proportion of sequences with more than one color in common sold by players with ( 94 players) and without (49 players) the Color Expert skill.

In Figure 5, we show the comparison of the proportion of multicolored sequences sold by players with the Color Expert skill and players without it. Interestingly, the median values for both groups are almost identical: 0.317 (or 31.7\%) for the players with the skill and 0.313 (or 31.3\%) for the players without the skill. The distribution of the proportion of multicolored sequences was not normal (the Shapiro-Wilk test rejected the null hypothesis with $p=0.3 E-4$ ), so we did a Mann-Whithney's U test to compare the medians. As expected, the test failed to reject the null hypothesis that the values were sampled from the same distribution $(p=0.89)$.

We conclude that the Color Expert skill does not affect the behavior of the players. This can be explained by the fact that one of the main goals of the game is to create sequences with as many colors in common as possible, whether the player selects this skill or not.

\section{Sequence Collector}

Every point in the Sequence Collector skill gives an additionnal slot to build a sequence. Because of the limited size of the player's hand and the limited number of sequence slots, it's hard to build long sequences with many colors in common. It is for both the sequence length and the number of colors in common that we added the Sequence Collector skill in the game.
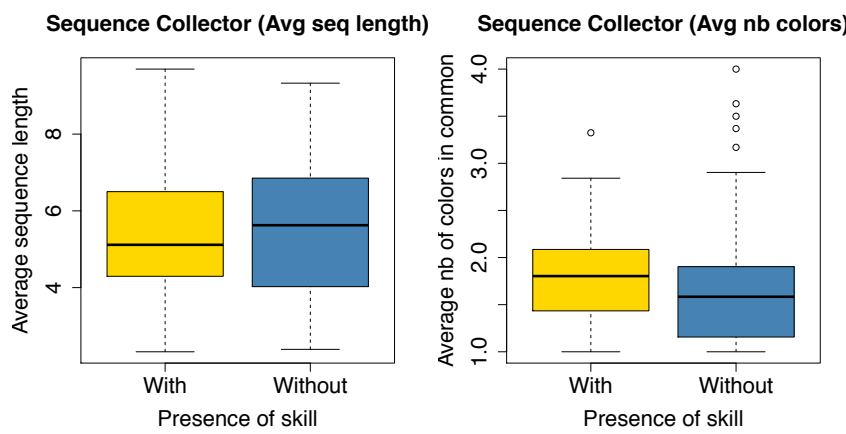

Figure 6. Boxplots of the average sequence length (left) and the average number of colors in common (right) of sequences built by players with (60 players) and without (83 players) the Sequence Collector skill.

We first compared the average sequence length of sequences built by players with the Sequence Collector skill and the ones built by the rest of players (see Figure 6-left). While the median value for the players without the skill (5.63) is a little bit larger than the one for the players with the skill (5.12), the averages of both groups are actually similar (5.61 and 5.56 in the same order). Since the distribution of the average sequence lengths were not normal (the Shapiro-Wilk test rejected the null hypothesis with $p=0.0057$ ), we did a Mann Whitney's $\mathrm{U}$ test to compare the medians of both groups. The test failed to reject the null hypothesis that the values were sampled from the same distribution $(p=0.69)$. Thus, there is no evidence that the Sequence Collector skill helps players build longer sequences. This tends to confirm what we mentioned earlier (in Section Testing hypothesis 1): the presence of the skills in general does not seem to affect the length of the sequences built by players. Once again, this can be explained by the fact that selling long sequences is one of the two main goals of the game, and is one of the main components of the scoring function.

We then compared the average number of colors in common of the sequences built by players with and without the Sequence collector skill (see Figure 6-right). The median value for the players without the skill (1.58) is $12 \%$ lower than the one for the players with the skill (1.80). Since the distribution of the average number of colors in common is not following a 
normal distribution (the Shapiro-Wilk test rejected the null hypothesis with $p=1.2 E-7$ ), we used a Mann-Whitney's U test to compare the medians of the two groups and we found a significant effect of the presence of this skill on the medians $(U=3113, p=0.01$, effect size $r=0.21)$. The Sequence collector skill is thus helping players to build sequences with more colors, by allowing them to store unfinished sequences of multiple colors in the additional slots until they are able to complete them.

\section{Testing hypothesis 3: the usefulness of the challenge system}

The challenge system was implemented to analyze the current state of the game and guide the players towards doing actions that are currently needed. As mentionned previously, five different challenge types were implemented in the game (see Section Challenge system for the complete list). In order to analyze the effect of the challenges on the way the participants were playing, for each challenge type, we compared the relevant statistics of the game during the challenge with the rest of the game session (when a different challenge was available).

Note that we are considering here only the nine sessions in which the challenges were present and that the Sell/buy and Buyout challenges were disabled during the session without the market.

\section{Minimum number of colors challenge}

To measure the effect of the Minimum number of colors challenge on the game, we compared the average number of colors of the sequences built by the players when the challenge was active and when it was not. The different averages for each game session are presented in Figure 7. In all the game sessions except A-3 and NM, the average number of colors in common is higher when the challenge is active.

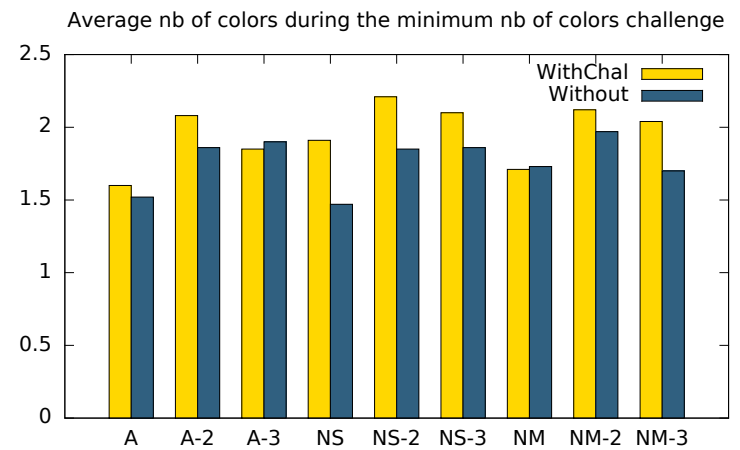

Figure 7. Average number of colors in the sequences with and without the Minimum number of colors challenge active. 'A', 'A-2' and 'A-3' represent the tests with all the features present, 'NS', 'NS-2' and 'NS3' represent the tests without the skills, and 'NM', 'NM-2' and 'NM-3' represents the tests without the market.

The distribution of the averages of the number of colors in common for all the game sessions considered here is normal (Shapiro-Wilk $p=0.79$ ), allowing us to use a Welch's t-test to compare the means for both groups, i.e. 1.96 colors in common during the challenge and 1.76 during the rest of the time. The test confirmed a significant effect of the presence of the challenge on the average number of colors in common $(t(16)=2.19, p=0.04$, Cohen's $d=1.03)$.

\section{Minimum sequence length challenge}

In order to analyze the effect that the Minimum sequence length challenge had on the game, we compared the average sequence length during the challenge and when a different challenge was active for all the game sessions. As shown in Figure 8, the presence of this challenge increased the average sequence length in all the game sessions except the three sessions with all the features.

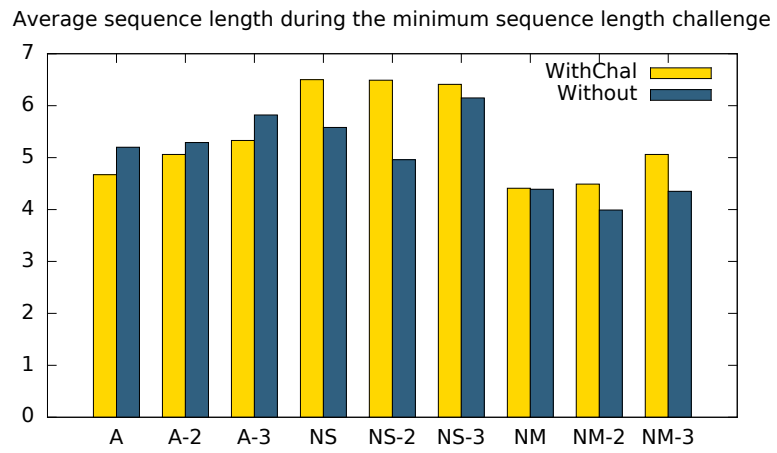

Figure 8. Average sequence length with and without the Minimum sequence length challenge active. 'A', 'A-2' and 'A-3' represent the tests with all the features present, 'NS', 'NS-2' and 'NS-3' represent the tests without the skills, and 'NM', 'NM-2' and 'NM-3' represents the tests without the market.

The means of all the average sequence lengths during the challenge and for the rest of the time are 5.38 and 5.08 respectively. Since the distribution of the averages of sequence lengths is normal (Shapiro-Wilk $p=0.27$ ), we used a Welch's t-test to compare those means, but the test wasn't able to prove that those means are significantly different $(t(16)=0.79$, $p=0.44)$.

Although there is not a statistically significant difference between the two groups, we can generally see a small effect for six of the nine groups with challenges. The fact that we observe the opposite effect in the three game sessions with all the features is very surprising, but hard to explain. One possible explanation could be that when all the features are present, the players have more to think about and check the challenges a little bit less.

\section{Sell/buy challenge}

For the Sell/buy challenge, we were interested in comparing the number of individual circles sold on the market per minute when the challenge was active and when it was not. The results, presented in Figure 9, don't show a clear trend. Indeed, in half of the game sessions, the number of circles sold per minute is higher during the challenge, while it's the opposite for the other half of the game sessions.

Once again, the numbers of circles sold per minute in the six different game sessions follow a normal distribution (ShapiroWilk $p=0.26$ ), so we used a Welch's t-test to compare the means of both groups, which are 13.18 during the challenge and 12.73 during the rest of the time. The t-test failed to reject 
Number of circles sold per minute during the sell/buy challenge

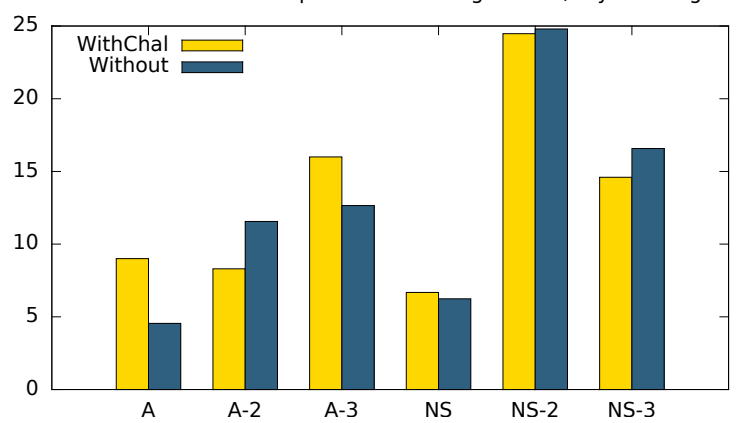

Figure 9. Number of individual circles sold on the market per minute with and without the Sell/buy challenge active. 'A', 'A-2' and 'A-3' represent the tests with all the features present, and 'NS', 'NS-2' and 'NS-3' represent the tests without the skills.

the null hypothesis that both means are the same $(t(10)=0.11$, $p=0.91)$.

We believe that the main reason why there doesn't seem to be any difference between the two groups is that most people were able to complete this type of challenge without really changing anything to their normal behavior. This challenge was simply too easy, because most of the players are always selling or buying (through the bids) at least 2 or 4 circles every five minutes (the length of a challenge).

\section{Buyout challenge}

The Buyout challenge appeared only once in total in all the three gaming session with challenges and with the market. Thus, we don't have a significant amount of data to analyze the effect of this challenge. The reason why this challenge almost never appeared is because players were always using the buyout, which greatly reduced the probability of showing this challenge.

\section{Specific colors in common challenge}

The Specific colors in common challenge is also difficult to analyze because it was completed only 8 times in total during the nine sessions with challenges, despite appearing 11 times throughout those nine experiments. This can be explained by the fact that it was the hardest challenge. All the other challenges are more general and can be completed by doing actions that are not specific to a certain subset of colors. Even if the market should be helpful in finding circles with the required subset of colors, it seems highly probable that the players felt that this type of challenge was too hard and almost never tried to complete it.

\section{Testing hypothesis 4: percentage of the problem solved} as a measure of the importance of different game features One of the research goals was to measure the impact of each feature by analyzing how much of the problem can be solved by the players in each of the game sessions. Our initial hypothesis was that players who have access to all the game features should be able to solve more of the problem.

Interestingly, we observed a larger than expected variance in the participants' personal skills which made it sometimes difficult to compare one game session with another in terms of the percentage of the problem that was solved. Indeed, some players quickly understood all the rules of the game and how to maximize their score, while others struggled to make points during the whole session, even with our help.

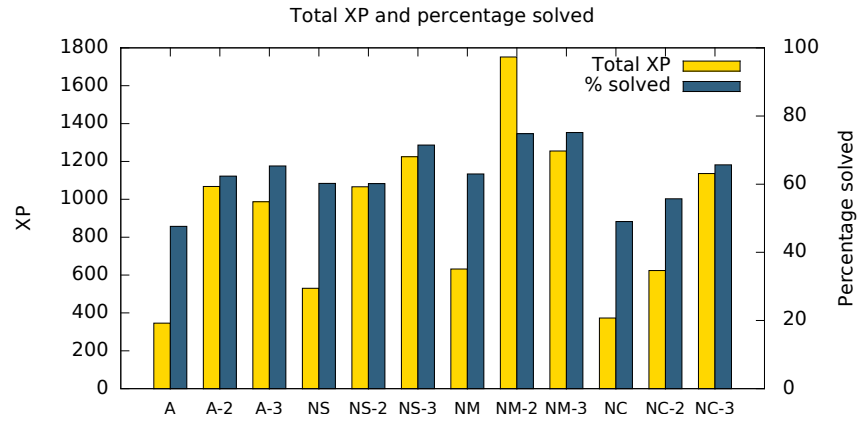

Figure 10. Total game experience and percentage of the problem solved for each of the 12 game sessions. 'XP' represents experience points. 'A', 'A-2' and 'A-3' represent the tests with all the features on; 'NS', 'NS2' and 'NS-3' represent the tests without the skills; 'NM', 'NM-2' and 'NM-3' represent the tests without the market; 'NC', 'NC-2' and 'NC-3' represent the tests without the challenges.

As shown in Figure 10, the percentage of the problem that was solved varies from $48 \%$ to $75 \%$ in all the different experiments. In particular, the differences observed for experiments with the exact same game conditions (sometimes up to a $18 \%$ difference) demonstrates that we cannot simply use the percentage of the exact solution found as a way to measure the impact of a feature. Moreover, the top five sessions in terms of percentage solved (all sessions with more than 65\%) come from the four different game conditions.

We used linear regression to test if the percentage of the problem solved is, to some extent, directly proportional to the total experience points accumulated by all the players during a session, which is a good way to measure the skills of the players during each session. The linear function obtained (graph not shown) had a coefficient of correlation $r=0.89$, which shows a strong level of correlation. Thus, it suggests that the participants' skill level has a greater effect on the size of the collected solutions than the game features alone.

\section{Understanding what makes a good player}

Based on the questionnaire filled by the players before playing the game, and the global leaderboard of all the players from all the sessions put together, we tried to find similarities between the top players. Table 3 shows the most interesting differences between the top 12 players and the rest of the players. In the questionnaires, players had to indicate their age category (between 21 and 25 for example), their own evaluation of their puzzle solving abilities and a range of hours spent playing video games every week.

The average age of the two groups of players was calculated by taking the middle point of the age categories. The average age of the top 12 players was about 2.5 years younger than the one of the other players. For the puzzle solving self evaluation, 
the players could choose a level between 1 and 5 ( 5 being the strongest). The average level of the top 12 players was 3.67, compared to 2.90 for the others. As we did with the age categories, we computed averages of time spent playing video games every week using the middle point of the categories. The top 12 players were playing roughly 2.5 times more every week than the rest of the players.

\begin{tabular}{ccc}
\hline & Top 12 players & Others \\
Age & 23.42 & 25.99 \\
Self evaluation (0-5) & 3.67 & 2.90 \\
Game time (hrs) & 10.00 & 4.11 \\
\hline
\end{tabular}

Table 3. Average statistics on the top 12 players vs the others.

We also looked at the mean age, mean video game time per week, and mean puzzle solving self evaluation for each of the 12 groups. We used linear regression to measure the correlation between those mean values and the total experience points gained by all the players of the groups. Mean age and mean video game time per week have a moderate level of correlation with total experience $(r=-0.67$ and $r=0.58$ respectively), while mean self evaluation only exhibits a weak level of correlation with total experience $(r=0.39)$.

\section{CONCLUSION}

We implemented a human computing game that uses a market, skills and challenges in order to solve a problem collaboratively. The problem that is solved by the players in our game is a graph problem that can be easily translated into a color matching game. The total number of colors used in the tests was small enough so that we were able to compute an exact solution and evaluate the performance of the players. We organized 12 game sessions of 10 players with four different game conditions (three times each).

Our tests showed that the market is a useful tool to help players build longer solutions (sequences, in our case). We also observed that weaker players can benefit from the market: it allows them to make larger contributions on average than stronger players without the market. In other words, the market allows participants of any skill level to be more productive. In addition, it also makes the game more dynamic and players mentioned that they really enjoyed this aspect of the game.

Our results also showed that skills in general are helpful to influence and guide the players into doing specific actions that are beneficial to the system and other players. We have found that skills are more efficient in their role of guiding the players if they are not directly related to the main goal of the game: the Color Expert skill for example did not affect the proportion of multicolored sequences built by the players.

The results on the challenges indicate that they can be useful to promote an action in the game (Minimum number of colors in common for example), but in order to be effective, the difficulty needs to be well-balanced. Challenges that are too easy (Sell/buy challenge for example) or too hard (Specific colors in common challenge for example) do not affect the game significantly.
Although the great variability in the participants' personal skills made it very difficult to make direct comparisons between the different game conditions in regards to the percentage of the solutions found, we showed that the percentage solved is proportional to the total experience gained by all players during a game session. Therefore, the percentage of the problem solved is clearly not only dependent on the features present in the game, but also on the participants' ability to be good at the game.

The analysis of the best players tends to show that younger players who play video games on a regular basis and have a strong self evaluation of their puzzle solving skills are able to understand the rules of the game and find winning strategies faster than the average participant.

In the context of a human computing game, where participants are contributing for free, players' perception and statisfaction with the gameplay is very important. We asked the players in the questionnaire to rate their experience with the game and the average score was $71.6 \%$, which is significantly above average. We also gathered some feedback when we were talking to the participants after the sessions. Since the game has no end in itself (we decided to limit the sessions to 45 minutes), some players mentioned that it is very addictive. Competing against other players to be at the top of the leaderboard was also a powerful motivation for the players. Obviously, we also had some negative comments: some players found the game hard to understand, while some very good players found it too easy. Overall, it is quite interesting to see that players enjoyed the game in its current state without any extensive effort put into visual aesthetics.

Another important point for a collaborative game like ours is scalability. In the tests that we have done during the development of the game, we have noticed that having more players is actually beneficial to the system. There are more sellers and buyers on the market, which results in a market that is a lot more dynamic (bidders get their circles faster, prices change more often, etc.). A more dynamic market allows different strategies to be equally profitable (e.g. some players can decide to focus on the Master Trader skill that boosts prices for selling circles, and then focus entirely on selling individual circles to other players). We are planning as future work to build an online version of the game that would be available $24 / 7$. This will allow us to analyze the differences in gameplay and quality of solutions between peak hours (with possibly thousands of players at the same time) and normal hours.

\section{ACKNOWLEDGMENTS}

First and foremost, the authors wish to thank all the players who made this study possible. The authors would also like to thank Jean-François Bourbeau, Mathieu Blanchette, Derek Ruths and Edward Newell for their help with the initial design of the game, and Alexandre Leblanc for his helpful advice on the statistical tests. The authors wish to thank Silvia Juliana Leon Mantilla and Shu Hayakawa for their help with the organization of the game sessions and the recruitment of participants. This work was made possible by grants from CIHR (BOP-130836), Genome Canada, NSERC (386596-10), and FRQNT. 


\section{REFERENCES}

1. Michael Buhrmester, Tracy Kwang, and Samuel D. Gosling. 2011. Amazon's Mechanical Turk: A New Source of Inexpensive, Yet High-Quality, Data? Perspectives on Psychological Science 6, 1 (2011), 3-5. DOI : http://dx. doi .org/10.1177/1745691610393980

2. Marija Mitrović Dankulov, Roderick Melnik, and Bosiljka Tadić. 2015. The dynamics of meaningful social interactions and the emergence of collective knowledge. Scientific Reports 5, 12197, Article 12197 (2015). DOI: http://dx.doi.org/10.1038/srep12197

3. Steven Dow, Anand Pramod Kulkarni, Scott R. Klemmer, and Björn Hartmann. 2012. Shepherding the crowd yields better work. In CSCW'12 Computer Supported Cooperative Work, Seattle, WA, USA, February 11-15, 2012. 1013-1022. DOI :

http://dx.doi .org/10.1145/2145204.2145355

4. Nathan Green, Paul Breimyer, Vinay Kumar, and Nagiza F. Samatova. 2010. PackPlay: Mining Semantic Data in Collaborative Games. In Proceedings of the Fourth Linguistic Annotation Workshop (LAW IV '10). Association for Computational Linguistics, Stroudsburg, PA, USA, 227-234.

http : //dl . acm . org/citation. cfm?id=1868720. 1868757

5. Andrea Guazzini, Daniele Vilone, Camillo Donati, Annalisa Nardi, and Zoran Levnajić. 2015. Modeling crowdsourcing as collective problem solving. Scientific Reports 5, 16557, Article 16557 (2015). DOI: http://dx.doi.org/10.1038/srep16557

6. Michiel Hildebrand, Maarten Brinkerink, Riste Gligorov, Martijn van Steenbergen, Johan Huijkman, and Johan Oomen. 2013. Waisda?: Video Labeling Game. In Proceedings of the 21st ACM International Conference on Multimedia (MM '13). ACM, New York, NY, USA, 823-826. DOI :

http: //dx.doi .org/10.1145/2502081.2502221

7. Chien-Ju Ho, Tsung-Hsiang Chang, and Jane Yung-jen Hsu. 2007. PhotoSlap: A Multi-player Online Game for Semantic Annotation. In Proceedings of the Twenty-Second AAAI Conference on Artificial Intelligence, July 22-26, 2007, Vancouver, British Columbia, Canada. 1359-1364.

http: //www . aaai . org/Library/AAAI/2007/aaai07-215 . php

8. Chien-Ju Ho, Tao-Hsuan Chang, Jong-Chuan Lee, Jane Yung-jen Hsu, and Kuan-Ta Chen. 2010. KissKissBan: A Competitive Human Computation Game for Image Annotation. SIGKDD Explor. Newsl. 12, 1 (Nov. 2010), 21-24. DOI : http://dx. doi . org/10.1145/1882471.1882475

9. Chang Hu, Benjamin B. Bederson, and Philip Resnik. 2010. Translation by Iterative Collaboration Between Monolingual Users. In Proceedings of the ACM SIGKDD Workshop on Human Computation (HCOMP '10). ACM, New York, NY, USA, 54-55. DOI :

http: //dx.doi.org/10.1145/1837885.1837902
10. Panagiotis G Ipeirotis and Evgeniy Gabrilovich. 2014. Quizz: targeted crowdsourcing with a billion (potential) users. In Proceedings of the 23rd international conference on World wide web. ACM, 143-154.

11. Geoff Kaufman, Mary Flanagan, and Sukdith Punjasthitkul. 2016. Investigating the Impact of'Emphasis Frames' and Social Loafing on Player Motivation and Performance in a Crowdsourcing Game. In Proceedings of the 2016 CHI Conference on Human Factors in Computing Systems. ACM, 4122-4128.

12. Alexander Kawrykow, Gary Roumanis, Alfred Kam, Daniel Kwak, Clarence Leung, Chu Wu, Eleyine Zarour, Phylo players, Luis Sarmenta, Mathieu Blanchette, and Jérôme Waldispühl. 2012. Phylo: a citizen science approach for improving multiple sequence alignment. PLoS One 7, 3 (2012), e31362. DOI:

http://dx.doi .org/10.1371/journal . pone. 0031362

13. Michael Kearns. 2012. Experiments in social computation. Commun. ACM 55, 10 (2012), 56-67. DOI : http://dx. doi . org/10.1145/2347736.2347753

14. Michael Kearns, Siddharth Suri, and Nick Montfort. 2006. An experimental study of the coloring problem on human subject networks. Science 313, 5788 (Aug 2006), 824-7. DOI: http://dx. doi.org/10.1126/science.1127207

15. Aniket Kittur and Robert E. Kraut. 2008. Harnessing the wisdom of crowds in wikipedia: quality through coordination. In Proceedings of the 2008 ACM Conference on Computer Supported Cooperative Work, CSCW 2008, San Diego, CA, USA, November 8-12, 2008. 37-46. DOI : http://dx. doi . org/10.1145/1460563.1460572

16. Markus Krause and René Kizilcec. 2015. To Play or Not to Play: Interactions between Response Quality and Task Complexity in Games and Paid Crowdsourcing. In Third AAAI Conference on Human Computation and Crowdsourcing.

17. Markus Krause, Aneta Takhtamysheva, Marion Wittstock, and Rainer Malaka. 2010. Frontiers of a Paradigm: Exploring Human Computation with Digital Games. In Proceedings of the ACM SIGKDD Workshop on Human Computation (HCOMP '10). ACM, New York, NY, USA, 22-25. DOI : http://dx. doi.org/10.1145/1837885.1837893

18. Daniel Kwak, Alfred Kam, David Becerra, Qikuan Zhou, Adam Hops, Eleyine Zarour, Arthur Kam, Luis Sarmenta, Mathieu Blanchette, and Jérôme Waldispühl. 2013. Open-Phylo: a customizable crowd-computing platform for multiple sequence alignment. Genome Biol 14, 10 (2013), R116. DOI :

http://dx.doi.org/10.1186/gb-2013-14-10-r116

19. Edith Law and Luis von Ahn. 2009. Input-agreement: A New Mechanism for Collecting Data Using Human Computation Games. In Proceedings of the SIGCHI Conference on Human Factors in Computing Systems (CHI '09). ACM, New York, NY, USA, 1197-1206. DOI : http://dx. doi .org/10.1145/1518701.1518881 
20. Greg Little. 2010. Exploring iterative and parallel human computation processes. In Proceedings of the 28th International Conference on Human Factors in Computing Systems, CHI 2010, Extended Abstracts Volume, Atlanta, Georgia, USA, April 10-15, 2010. 4309-4314. DOI :

http://dx. doi .org/10.1145/1753846.1754145

21. Daniel Lombrana, Marvin Reimer, Alejandro Dominguez, James Doherty, Jorge Correa, Clara Sanchez-Puga, and Alvaro Suarez Perez. 2015. http://crowdcrafting.org/. (2015). http://crowdcrafting . org/

22. Jan Lorenz, Heiko Rauhut, Frank Schweitzer, and Dirk Helbing. 2011. How social influence can undermine the wisdom of crowd effect. Proc Natl Acad Sci U S A 108, 22 (May 2011), 9020-5. DOI : http://dx. doi . org/10.1073/pnas. 1008636108

23. Koji Oishi, Manuel Cebrian, Andres Abeliuk, and Naoki Masuda. 2014. Iterated crowdsourcing dilemma game. Scientific Reports 4, 4100, Article 4100 (2014). DOI : http: //dx. doi . org/10.1038/srep04100

24. Gabriele Paolacci, Jesse Chandler, and Panagiotis G. Ipeirotis. 2010. Running Experiments on Amazon Mechanical Turk. Judgment and Decision Making 5, 5 (2010), 411-419.

25. Massimo Poesio, Jon Chamberlain, Udo Kruschwitz, Livio Robaldo, and Luca Ducceschi. 2013. Phrase Detectives: Utilizing Collective Intelligence for Internet-scale Language Resource Creation. ACM Trans. Interact. Intell. Syst. 3, 1, Article 3 (April 2013), 44 pages. DOI : http://dx. doi . org/10.1145/2448116.2448119

26. Jimmy Secretan, Nicholas Beato, David B. D Ambrosio, Adelein Rodriguez, Adam Campbell, and Kenneth O. Stanley. 2008. Picbreeder: Evolving Pictures Collaboratively Online. In Proceedings of the SIGCHI Conference on Human Factors in Computing Systems (CHI '08). ACM, New York, NY, USA, 1759-1768. D0I : http://dx. doi .org/10.1145/1357054.1357328
27. Luis von Ahn and Laura Dabbish. 2004. Labeling images with a computer game. In Proceedings of the 2004 Conference on Human Factors in Computing Systems, CHI 2004, Vienna, Austria, April 24 - 29, 2004. 319-326. DOI : http://dx.doi . org/10.1145/985692.985733

28. Luis von Ahn, Ruoran Liu, and Manuel Blum. 2006. Peekaboom: A Game for Locating Objects in Images. In Proceedings of the SIGCHI Conference on Human Factors in Computing Systems (CHI '06). ACM, New York, NY, USA, 55-64. DOI :

http://dx. doi .org/10.1145/1124772.1124782

29. Borui Wang and Jingshu Chen. 2013. Visimu: A Game for Music Color Label Collection. In Proceedings of the Adjunct Publication of the 26th Annual ACM Symposium on User Interface Software and Technology (UIST'13 Adjunct). ACM, New York, NY, USA, 93-94. DOI : http://dx. doi . org/10.1145/2508468.2514726

30. Lixiu Yu and Jeffrey V. Nickerson. 2011. Cooks or cobblers?: crowd creativity through combination. In Proceedings of the International Conference on Human Factors in Computing Systems, CHI 2011, Vancouver, BC, Canada, May 7-12, 2011. 1393-1402. D0I: http://dx. doi . org/10.1145/1978942 . 1979147

31. Haoqi Zhang, Edith Law, Rob Miller, Krzysztof Gajos, David C. Parkes, and Eric Horvitz. 2012. Human computation tasks with global constraints. In $\mathrm{CHI}$ Conference on Human Factors in Computing Systems, CHI '12, Austin, TX, USA - May 05 - 10, 2012. 217-226. DOI : http://dx.doi .org/10.1145/2207676.2207708 